\title{
Robust Stability/Performance Analysis for Linear Time-Invariant Polynomially Parameter-Dependent Systems Using Polynomially Parameter-Dependent Lyapunov Functions
}

\author{
Masayuki SATo* and Dimitri Peaucelle**
}

\begin{abstract}
This paper addresses robust stability, $H_{2}$ performance, and $H_{\infty}$ performance analysis for Linear Time-Invariant Parameter-Dependent (LTIPD) systems using Parameter-Dependent Lyapunov Functions (PDLFs). The statespace matrices of the LTIPD systems are set to be parameter-dependent in negative as well as positive power series with respect to the parameters, and PDLFs are also set to be parameter-dependent in negative as well as positive power series with respect to the parameters. Our results are derived using "slack variables" and restricting them to be parameter-independent, therefore, they are only sufficient conditions for the original analysis problems. However, we demonstrate the effectiveness of our results with randomly generated numerical examples and several numerical examples which are borrowed from existing papers.
\end{abstract}

Key Words: robust stability, robust performance, linear time-invariant parameter-dependent systems, polynomially parameter-dependent systems.

\section{Introduction}

Since de Oliveira et al. proposed a new robust stability analysis method for Linear Time-Invariant ParameterDependent (LTIPD) systems using slack variables ${ }^{1)}$, much research using slack variables has been conducted for this decade; robust stability analysis ${ }^{2) \sim 6)}$, robust $D$-stability analysis ${ }^{7)}$, robust $H_{2}$ performance analysis $^{5), 8), 9)}$, and robust $H_{\infty}$ performance analysis ${ }^{4) \sim 6)}$. All their derived conditions are sufficient conditions for the original analysis problems because slack variables need to be parameter-independent to derive parametrically affine Linear Matrix Inequalities (LMIs). To reduce the conservatism, new methods using parameter-dependent slack variables have been proposed ${ }^{10) \sim 14)}$, some of which formulate sufficient conditions which asymptotically tend to be the necessary conditions if the parameter-dependency of Lyapunov functions is given a priori. Several other approaches for robust stability analysis and robust performance analysis have been also proposed; quadratic separator approach ${ }^{15), 16)}$, sums-of-squares approach ${ }^{17), 18)}$, and KYP lemma approach ${ }^{19)}$, some of which formulate the necessary and sufficient conditions for the original analysis problems if the parameter-dependency of Lyapunov functions is given a priori. Compared to

* Japan Aerospace Exploration Agency, Mitaka

** LAAS-CNRS, University of Toulouse, France

(Received September 19, 2006)

(Revised April 8, 2007) these methods, simple slack variable approach (not using parameter-dependent slack variables) has the drawback that derived conditions are only sufficient conditions for the original problems even if the parameter-dependency of Lyapunov functions is increased.

Most of existing papers for robust stability/performance analysis (except 2), 5), and 20), the last considers more general nonlinear models) tackle the stability/performance analysis for polytopically parameterdependent systems. However, the representation of polytopically parameter-dependent systems sometimes leads to increased conservatism. Let consider the system defined as $\dot{x}=\left[\begin{array}{cc}-(\theta+1 / \theta) & 1 \\ 1 & -\theta\end{array}\right] x$, where $x$ is the state vector and $\theta\left(0<\delta_{\min } \leq \theta \leq \delta_{\max }\right)$ is a parameter representing the uncertainty. To treat this system as a polytopic system, we first make a polytope which covers the variation of the system matrix; that is, make a polytope which covers the variation of $\theta+1 / \theta$, e.g. a polytope with three vertices $A, B$ and $C$ or a polytope with four vertices $A, B, D$ and $E$ in Fig. 1. Although we can obtain tighter polytopes than these, they always contain the region between $\theta+1 / \theta$ and line $A B$ which are depicted as a gray region in Fig. 1, which leads to conservatism because we have no need to cover it (this will be shown in Section 5). As another method, we can apply biquadratic stability proposed in 3 ) in the following sequence; we first set the number of parameters in this system to be two; $\theta$ and $\alpha:=1 / \theta$ because biquadratic stability is applicable only for parametrically affine systems, then we apply bi- 


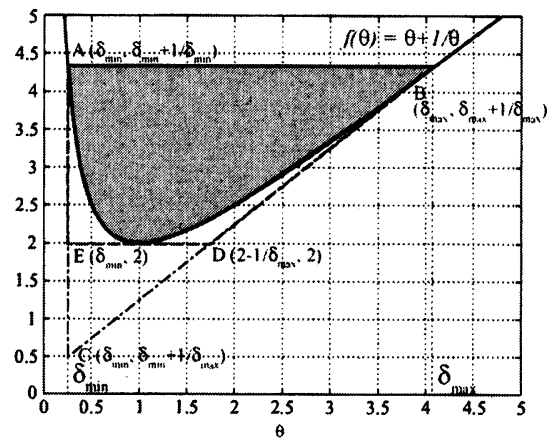

Fig. 1 Polytopic covers for $\theta+1 / \theta$

quadratic stability to this modified system. However, generally speaking, such modification leads to conservatism, because such modification neglects the relationship between $\theta$ and $1 / \theta$ (this will be also shown in Section 5). Similarly, in 2), the authors have proposed another analysis method for polynomially parameter-dependent systems. However, they also use quadratically parameterdependent Lyapunov functions, which subsequently leads to conservative analysis. In contrast to these methods, in 5), the author has proposed an analysis method applicable for polynomially parameter-dependent systems, which theoretically encompasses biquadratic stability in 3). However, the formulation has the drawback that LTIPD systems cannot have terms with more than three parameters multiplied. This restriction is removed in the present manuscript.

In this paper, we address robust stability, robust $\mathrm{H}_{2}$ performance, and $H_{\infty}$ performance analysis for LTIPD systems, in which the state-space matrices are parameterdependent in negative as well as positive power series of parameters and all their by-products. Results are derived using Lyapunov functions which are parameter-dependent in negative as well as positive power series with respect to parameters and all their by-products. We adopt slack variable approach, therefore, our results are only sufficient conditions for the original analysis problems. However, we demonstrate the effectiveness of our methods with randomly generated examples and several numerical examples borrowed from existing papers. In other words, we demonstrate that simple slack variable approach is not so conservative compared to existing methods. The main contribution of this paper is to propose the general formulations using polynomially Parameter-Dependent Lyapunov Functions (PDLFs) not only for robust stability analysis but also for performance analysis for LTIPD systems via simple slack variable approach, in which the increased-order PDLFs lead to decreased conservatism is guaranteed.

Hereafter, $\mathrm{He}(X)$ is the shorthand notation of $X+X^{T}$, $0_{n}, 0_{n, m}, I_{n}$ and $\mathbf{0}$ respectively denote an $n$-dimensional square zero matrix, an $n \times m$-dimensional zero matrix, an $n$-dimensional identity matrix and an appropriately dimensional zero matrix, $\mathcal{R}^{n \times m}$ and $\mathcal{S}^{n}$ respectively denote sets of $n \times m$ dimensional real matrices and $n \times n$ dimensional symmetric real matrices, $\otimes$ denotes Kronecker product, $X^{\perp} \in \mathcal{R}^{(n-r) \times n}$ denotes a matrix satisfying $X^{\perp} X=\mathbf{0}$ and $X^{\perp} X^{\perp^{T}}>0$, where $X \in \mathcal{R}^{n \times p}$ and $\operatorname{rank}(X)=r$, and the notation $\operatorname{Tr}_{n}(X)$ denotes $\left[\begin{array}{ccc}\operatorname{Tr}\left(X_{11}\right) & \cdots & \operatorname{Tr}\left(X_{1 i}\right) \\ \vdots & \ddots & \vdots \\ \operatorname{Tr}\left(X_{i 1}\right) & \cdots & \operatorname{Tr}\left(X_{i i}\right)\end{array}\right]$ for $X=\left[\begin{array}{ccc}X_{11} & \cdots & X_{1 i} \\ \vdots & \ddots & \vdots \\ X_{i 1} & \cdots & X_{i i}\end{array}\right]$, where $X_{k l} \in \mathcal{R}^{n \times n}(k, l=1, \cdots, i)$.

\section{Preliminaries}

In this section, we make some preliminaries for our proposed methods.

\section{1 Definitions}

In this paper, we consider the following LTIPD system.

$$
G(\theta):\left\{\begin{array}{l}
\dot{x}=A(\theta) x+B(\theta) w \\
z=C(\theta) x+D(\theta) w
\end{array},\right.
$$

where $x \in \mathcal{R}^{n}$ is the state vector, $w \in \mathcal{R}^{n_{w}}$ is the disturbance input vector, $z \in \mathcal{R}^{n_{z}}$ is the performance output vector, and matrices $A(\theta), B(\theta)$, etc. are parameterdependent matrices of appropriate dimensions. We assume that the LTIPD system has $k$ independent scalar parameters $\theta=\left[\begin{array}{lll}\theta_{1} & \cdots & \theta_{k}\end{array}\right]^{T}$. They represent time-invariant plant uncertainties and are supposed to lie in a convex hull with $N$ vertices $\theta \in \operatorname{co}\left\{\theta^{v_{1}} \ldots \theta^{v_{N}}\right\}$. For each parameter $\theta_{i}(i=1, \cdots, k)$, define the vector of its negative and positive power series ranging from $-l_{i}$ to $m_{i}$ as

$$
\theta_{i}^{\left[-l_{i}, m_{i}\right]}=\left[\begin{array}{lllllll}
\theta_{i}^{-l_{i}} & \cdots & \theta_{i}^{-1} & 1 & \theta_{i} & \cdots & \theta_{i}^{m_{i}}
\end{array}\right]^{T} \in \mathcal{R}^{\sigma_{i}},
$$

where $\sigma_{i}=l_{i}+m_{i}+1$. In the case that $l_{i} \neq 0$, we assume that $\theta_{i}^{-1}$ is well-defined, e.g. in the case that $l_{i} \neq 0(i=$ $1, \cdots, k)$, co $\left\{\theta^{v_{1}} \cdots \theta^{v_{N}}\right\}$ does not include the origin. Define the vector of all monomials obtained as products of all $\theta_{i}^{j}$ elements with $i=1, \cdots, k, j=-l_{i}, \cdots, m_{i}$ :

$$
\bar{\theta}=\theta_{1}^{\left[-l_{1}, m_{1}\right]} \otimes \cdots \otimes \theta_{k}^{\left[-l_{k}, m_{k}\right]} \in \mathcal{R}^{\pi^{[1, k]}},
$$

where $\pi^{[q, k]}=\Pi_{i=q}^{k} \sigma_{i}$. By definition, let $\pi^{[k+1, k]}=1$.

Based on these definitions, the parameter-dependent system is modeled as

$$
\begin{aligned}
& A(\theta)=\bar{A} \bar{\theta}_{n}, \quad \bar{A} \in \mathcal{R}^{n \times n \pi^{[1, k]}}, \\
& B(\theta)=\bar{B} \bar{\theta}_{n_{w}}, \bar{B} \in \mathcal{R}^{n \times n_{w} \pi^{[1, k]}}, \\
& C(\theta)=\bar{C} \bar{\theta}_{n}, \quad \bar{C} \in \mathcal{R}^{n_{z} \times n \pi^{[1, k]}}, \\
& D(\theta)=\bar{D} \bar{\theta}_{n_{w}}, \bar{D} \in \mathcal{R}^{n_{z} \times n_{w} \pi^{[1, k]}},
\end{aligned}
$$


where $\bar{\theta}_{n}=\bar{\theta} \otimes I_{n}$ and $\bar{\theta}_{n_{w}}=\bar{\theta} \otimes I_{n_{w}}$. Such representation models any power series from $-l_{i}$ to $m_{i}$ of the parameters $\theta_{i}(i=1, \cdots, k)$, and all their by-products. The matrices $\bar{A}, \bar{B}$, etc. are row-block matrices, each of which represent coefficients associated with one monomial of $\bar{\theta}$.

For this LTIPD system, we will look for a quadratic PDLF $x^{T} P(\theta) x$ with

$$
P(\theta)=\bar{P} \bar{\theta}_{n}=\bar{\theta}_{n}^{T} \bar{P}^{T}, \bar{P} \in \mathcal{R}^{n \times n \pi^{[1, k]}},
$$

where $\bar{P}$ is a row-block matrix of symmetric matrices.

Remark 1 Note that taking the $l_{i}$ and $m_{i}$ integers larger than needed for modeling the system (i.e. by adding zero matrices in the relevant positions of $\bar{A}, \bar{B}$, etc.), the $P D L F$ can be of higher-order than the model. Doing so reduces the conservatism of the following exposed results. This will be shown with several numerical examples in section 5.

Two additional notations are as well needed. Let the vector

$$
e=[\underbrace{0 \cdots 0}_{\sum_{i=1}^{k}\left(l_{i} \times \pi^{[i+1, k]}\right)} 1 \underbrace{0 \cdots 0}_{\sum_{i=1}^{k}\left(m_{i} \times \pi^{[i+1, k]}\right)}]^{T} \in \mathcal{R}^{\pi^{[1, k]}}
$$

and let $e_{n}=e \otimes I_{n}$. This notation works as an index for getting parameter-independent elements and gives the symmetrized version of $P(\theta)$ in (3):

$$
P(\theta)=\frac{1}{2} \bar{\theta}_{n}^{T}\left(\bar{P}^{T} e_{n}^{T}+e_{n} \bar{P}\right) \bar{\theta}_{n} .
$$

Let as well the affine parameter-dependent matrices $(i=$ $1, \cdots, k)$

$$
\eta_{i}(\theta)=\left[\begin{array}{c}
\theta_{i} I_{\sigma_{i}-1} \\
\mathbf{0}
\end{array}\right]-\left[\begin{array}{c}
\mathbf{0} \\
I_{\sigma_{i}-1}
\end{array}\right] \in \mathcal{R}^{\sigma_{i} \times\left(\sigma_{i}-1\right)}
$$

which satisfy $\eta_{i}(\theta)^{\perp}=\theta_{i}^{\left[-l_{i}, m_{i}\right]^{T}}$. At last, define

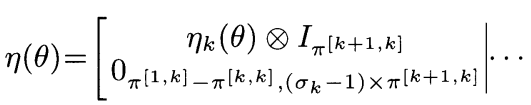

$$
\begin{aligned}
& \left.\left|\begin{array}{c}
\eta_{2}(\theta) \otimes I_{\pi^{[3, k]}} \\
0_{\left.\pi^{[1, k]}-\pi^{[2, k]},\left(\sigma_{2}-1\right) \times \pi^{[3, k}\right]}
\end{array}\right| \eta_{1}(\theta) \otimes I_{\pi^{[2, k]}}\right] \\
& \in \mathcal{R}^{\pi^{[1, k]} \times\left(\pi^{[1, k]}-1\right)},
\end{aligned}
$$

$\eta_{n}(\theta)=\eta(\theta) \otimes I_{n}$ and $\eta_{n_{w}}(\theta)=\eta(\theta) \otimes I_{n_{w}}$, which prove to satisfy $\eta(\theta)^{\perp}=\bar{\theta}^{T}, \eta_{n}(\theta)^{\perp}=\bar{\theta}_{n}^{T}$ and $\eta_{n_{w}}(\theta)^{\perp}=\bar{\theta}_{n_{w}}^{T}$.

\subsection{Basic Lemmas}

Well-known stability, $H_{2}$ performance, and $H_{\infty}$ performance analysis conditions for the LTIPD system (1) are now recalled. In these lemmas, Lyapunov functions are set to be $x^{T} P(\theta) x$.

Lemma 1 (Stability) The system (1) is robustly stable for all admissible $\theta$ if and only if there exists $P(\theta)>0$ that satisfies (4) for all admissible $\theta$.

$$
\mathrm{He}\{P(\theta) A(\theta)\}<0
$$

Lemma 2 ( $H_{2}$ performance) The system (1) is robustly stable and its $\mathrm{H}_{2}$ performance is bounded by $\gamma_{2}$ for all admissible $\theta$ if and only if $D(\theta)=\mathbf{0}$ and there exist $P(\theta)>0$ and $N(\theta)>0$ that satisfy (5), (6), and (7) for all admissible $\theta$.

$$
\begin{array}{r}
\text { He }\{P(\theta) A(\theta)\}+C(\theta)^{T} C(\theta)<0 \\
N(\theta)-B(\theta)^{T} P(\theta) B(\theta)>0 \\
\gamma_{2}^{2}>\operatorname{Tr}\{N(\theta)\}
\end{array}
$$

Lemma 3 ( $H_{\infty}$ performance) The system (1) is robustly stable and its $H_{\infty}$ performance is bounded by $\gamma_{\infty}$ for all admissible $\theta$ if and only if there exists $P(\theta)>0$ that satisfies (8) for all admissible $\theta$.

$$
\begin{aligned}
& {\left[\begin{array}{cc}
\operatorname{He}\{P(\theta) A(\theta)\} P(\theta) B(\theta) \\
B(\theta)^{T} P(\theta)^{T} & -\gamma_{\infty}^{2} I_{n_{w}}
\end{array}\right]} \\
& +\left[\begin{array}{l}
C(\theta)^{T} \\
D(\theta)^{T}
\end{array}\right][C(\theta) D(\theta)]<0
\end{aligned}
$$

In Lemmas 1,2 , and 3 , the positivity of $P(\theta)$ is redundant if the nominal system of (1) is stable ${ }^{9), 21)}$, which can be assumed without loss of generality. Thus, we do not consider the positivity condition of $P(\theta)$ hereafter.

\section{Main Results}

In this section, we show our proposed methods for stability analysis using Lemma $1, H_{2}$ performance analysis using Lemma 2 , and $H_{\infty}$ performance analysis using Lemma 3 for LTIPD system (1) using PDLF defined in (3).

\section{1 Basic Results}

We first show our basic results for stability and performance analysis.

Theorem 1 (Stability Analysis) If there exist matrices $\bar{P} \in \mathcal{R}^{n \times n \pi^{[1, k]}}$ and $M \in \mathcal{R}^{n\left(\pi^{[1, k]}-1\right) \times n \pi^{[1, k]}}$ such that

$$
\mathrm{He}\left\{\bar{P}^{T} \bar{A}+\eta_{n}(\theta) M\right\}<0
$$

holds for all vertices $\theta=\theta^{v_{1}}, \cdots, \theta^{v_{N}}$, then the LTIPD system (1) is robustly stable.

Proof 1 First note that (9) is affine with respect to $\theta$. Therefore if it holds for all vertices then it also holds for

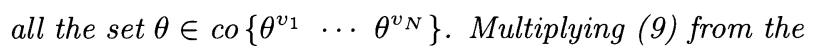


left and the right by $\bar{\theta}_{n}^{T}$ and $\bar{\theta}_{n}$ respectively leads to (4) directly. This completes the proof.

Remark 2 Note that the last operation corresponds to the elimination lemma ${ }^{22)}$, which is not lossless in this case since $M$ is assumed to be independent of $\theta$.

Theorem 2 ( $H_{2}$ Performance Analysis) If there exist a symmetric matrix $\bar{N} \in \mathcal{S}^{n_{w} \pi^{[1, k]}}$, matrices $\bar{P} \in \mathcal{R}^{n \times n \pi^{[1, k]}}, \quad F \in \mathcal{R}^{n\left(\pi^{[1, k]}-1\right) \times n \pi^{[1, k]}}, M \in$ $\mathcal{R}\left\{n_{w}\left(\pi^{[1, k]}-1\right)+n\left(\pi^{[1, k]}-1\right)\right\} \times\left(n_{w} \pi^{[1, k]}+n \pi^{[1, k]}\right)$ and $H \in$ $\mathcal{R}^{\left(\pi^{[1, k]}-1\right) \times \pi^{[1, k]}}$, and a positive number $\gamma_{2}$ such that

$$
\begin{aligned}
& \mathrm{He}\left\{\bar{P}^{T} \bar{A}+\eta_{n}(\theta) F\right\}+\bar{C}^{T} \bar{C}<0, \\
& {\left[\begin{array}{cc}
\bar{N} & \bar{B}^{T} \bar{P} \\
\bar{P}^{T} \bar{B} & \frac{\bar{P}^{T} e_{n}^{T}+e_{n} \bar{P}}{2}
\end{array}\right]} \\
& +\mathrm{He}\left(\left[\begin{array}{cc}
\eta_{n_{w}}^{2}(\theta) & \mathbf{0} \\
\mathbf{0} & \eta_{n}(\theta)
\end{array}\right] M\right)>0, \\
& \gamma_{2}^{2} e e^{T}-\operatorname{Tr}_{n_{w}}(\bar{N})+\operatorname{He}\{\eta(\theta) H\}>0
\end{aligned}
$$

hold for all vertices $\theta=\theta^{v_{1}}, \cdots, \theta^{v_{N}}$, then the LTIPD system (1) is robustly stable and an upper bound of its $\mathrm{H}_{2}$ performance is given by $\gamma_{2}$.

Proof of Theorem 2 is much alike that of Theorem 1 when choosing $N(\theta)=\bar{\theta}_{n_{w}}^{T} \bar{N} \bar{\theta}_{n_{w}}$, thus we omit it.

Theorem 3 ( $H_{\infty}$ Performance Analysis) If there exist matrices $\bar{P} \in \mathcal{R}^{n \times n \pi^{[1, k]}}$ and $\quad M \in$ $\mathcal{R}\left\{n\left(\pi^{[1, k]}-1\right)+n_{w}\left(\pi^{[1, k]}-1\right)\right\} \times\left(n \pi^{[1, k]}+n_{w} \pi^{[1, k]}\right)$, and a positive number $\gamma_{\infty}$ such that

$$
\begin{aligned}
& {\left[\begin{array}{cc}
\mathrm{He}\left(\bar{P}^{T} \bar{A}\right) & \bar{P}^{T} \bar{B} \\
\bar{B}^{T} \bar{P} & -\gamma_{\infty}^{2}\left(e e^{T} \otimes I_{n_{w}}\right)
\end{array}\right]+\left[\begin{array}{c}
\bar{C}^{T} \\
\bar{D}^{T}
\end{array}\right][\bar{C} \bar{D}]} \\
& +\operatorname{He}\left(\left[\begin{array}{cc}
\eta_{n}(\theta) & \mathbf{0} \\
\mathbf{0} & \eta_{n_{w}}(\theta)
\end{array}\right] M\right)<0
\end{aligned}
$$

holds for all vertices $\theta=\theta^{v_{1}}, \cdots, \theta^{v_{N}}$, then the LTIPD system (1) is robustly stable and an upper bound of its $H_{\infty}$ performance is given by $\gamma_{\infty}$.

Proof of Theorem 3 is much alike that of Theorem 1, thus we omit it.

\section{2 Less Conservative Conditions}

In the previous subsection, Lyapunov functions $P(\theta)$ are set to be as in (3). If we reduce the conservatism due to setting $P(\theta)$ to be in (3), increasing the parameterdependency of $P(\theta)$, which was indicated in Remark 1, is effective. However, it leads to increased numerical complexity because the sizes of additionally introduced matrices $M$, etc. are increased.

In this subsection, we show another methodology to tackle the conservatism due to setting $P(\theta)$ to be in (3). We now set $P(\theta)$ to be as follows.

$$
P(\theta)=\bar{P}(\theta) \bar{\theta}_{n}
$$

where $P(\theta)$ has the same definition as in (3) but with $\bar{P}(\theta)$ being chosen as an affine function of $\theta$. This choice increases by one the power of the PDLF.

Remark 3 If $\bar{P}(\theta)$ is set to be parameter-independent, then $P(\theta)$ in (14) is the same as $P(\theta)$ in (3). Therefore, using $P(\theta)$ in (14) is no more conservative than using $P(\theta)$ in (3).

Using $P(\theta)$ defined in (14), we obtain the following theorem.

Theorem 4 (Stability Analysis) If there exist a parametrically affine matrix $\bar{P}(\theta)$ and a matrix $M$ such that

$$
\mathrm{He}\left\{\bar{P}(\theta)^{T} \bar{A}+\eta_{n}(\theta) M\right\}<0,
$$

holds for all vertices $\theta=\theta^{v_{1}}, \cdots, \theta^{v_{N}}$, then the LTIPD system (1) is robustly stable.

Proof 2 Inequality (15) is affine with respect to $\theta$. Therefore if it holds for all vertices then it also holds for all the

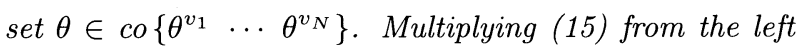
and the right by $\bar{\theta}_{n}^{T}$ and $\bar{\theta}_{n}$ respectively leads to (4) directly. This completes the proof.

We obtain the counterparts of Theorems 2 and 3 .

Theorem 5 ( $\mathrm{H}_{2}$ Performance Analysis) If there exist a symmetric parametrically affine matrix $\bar{N}(\theta)$, a parametrically affine matrix $\bar{P}(\theta)$, matrices $F, M$ and $H$, and a positive number $\gamma_{2}$ such that (10), (11) and (12), in which $\bar{P}$ and $\bar{N}$ are replaced by $\bar{P}(\theta)$ and $\bar{N}(\theta)$ respectively, hold for all vertices $\theta=\theta^{v_{1}}, \cdots, \theta^{v_{N}}$, then the LTIPD system (1) is robustly stable and an upper bound of its $\mathrm{H}_{2}$ performance is given by $\gamma_{2}$.

Proof of Theorem 5 is much alike that of Theorem 4 when choosing $N(\theta)=\bar{\theta}_{n_{w}}^{T} \bar{N}(\theta) \bar{\theta}_{n_{w}}$, where $\bar{N}(\theta)$ is an affine function of $\theta$. Thus we omit it.

Remark 4 If $\bar{P}(\theta)$ and $\bar{N}(\theta)$ are set to be parameterindependent, then Theorem 5 becomes Theorem 2. Therefore, Theorem 5 is no more conservative than Theorem 2.

Theorem 6 ( $H_{\infty}$ Performance Analysis) If there exist a parametrically affine matrix $\bar{P}(\theta)$, a matrix $M$, and a positive number $\gamma_{\infty}$ such that (13), in which $\bar{P}$ is replaced by $\bar{P}(\theta)$, holds for all vertices $\theta=\theta^{v_{1}}, \cdots, \theta^{v_{N}}$, then the LTIPD system (1) is robustly stable and an upper bound of its $H_{\infty}$ performance is given by $\gamma_{\infty}$. 
Proof of Theorem 6 is much alike that of Theorem 4, thus we omit it.

\section{Observations}

In this section, we first show that our stability analysis Theorem 1 encompasses the stability analysis based on quadratic stability for parametrically affine LTIPD systems. We next show that increasing parameterdependency of Lyapunov functions leads to less conservative analysis in exchange for increased numerical complexity. We have obtained similar assertions for $H_{2}$ performance and $H_{\infty}$ performance analysis, however, we omit them due to lack of space.

\section{1 Inclusion Relation}

In this subsection, we show that Theorem 1 encompasses quadratic stability. For simplicity, we assume that the number of parameters $k$ is three. However, we can easily extend the following result to the general case.

We now consider parametrically affine system for (1). Under this assumption, $A(\theta)$ in (2) is given as follows.

$$
A(\theta)=A_{0}+\Sigma_{i=1}^{3} \theta_{i} A_{i}=\bar{A} \bar{\theta}_{n},
$$

where $\bar{A}=\left[\begin{array}{llllll}A_{0} & A_{3} & A_{2} & 0_{n} & A_{1} & 0_{n, 3 n}\end{array}\right]$.

We give the following assertion.

Proposition 1 If there exists a symmetric matrix $P_{0} \in$ $\mathcal{S}^{n}$ such that $\mathrm{He}\left\{P_{0} A(\theta)\right\}<0$ holds for all vertices $\theta=\theta^{v_{1}}, \cdots, \theta^{v_{N}}$, then there always exist matrices $\bar{P}$ and $M$ such that (9) holds for all vertices $\theta=\theta^{v_{1}}, \cdots, \theta^{v_{N}}$.

Proof 3 We set $\bar{P}$ in Theorem 1 to be $\left[P_{0} 0_{n, 7 n}\right]$, and set $M$ in Theorem 1 to be as follows with sufficiently small positive scalars $\varepsilon_{1}, \varepsilon_{2}$ and $\varepsilon_{3}$.

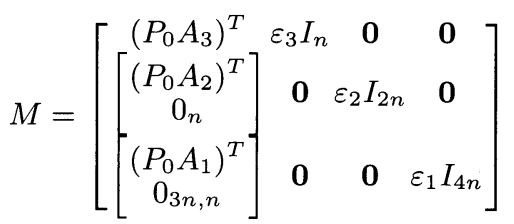

In the following, we obtain the equivalent condition to (9). We first obtain the following inequalities after applying Schur complement to (9).

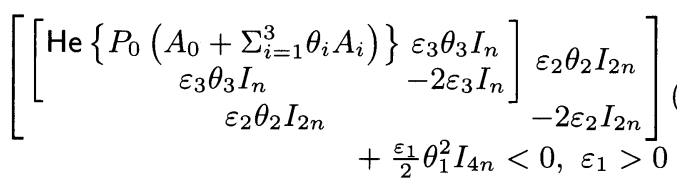

As the variation of $\theta_{1}$ is bounded, (17) always hold with sufficiently small positive scalar $\varepsilon_{1}$ if the first term of the first inequality is negative definite. We next consider the following inequality instead of the first one in (17).

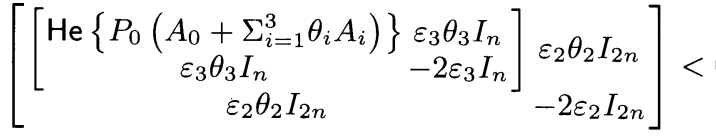

After applying Schur complement to (18), we then obtain the following inequalities.

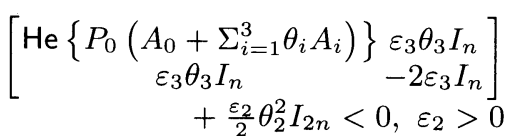

As the variation of $\theta_{2}$ is bounded, the above inequalities always hold with sufficiently small positive scalar $\varepsilon_{2}$ if the first term of the first inequality is negative definite. After applying the same procedure, which is used in the transformation from (17) to (18), to the first inequality in (19), we obtain the following inequalities.

$$
\mathrm{He}\left\{P_{0}\left(A_{0}+\Sigma_{i=1}^{3} \theta_{i} A_{i}\right)\right\}<0, \varepsilon_{3}>0
$$

Considering (17), (19) and (20), if there exist $P_{0}$ such that $\mathrm{He}\left\{P_{0}\left(A_{0}+\Sigma_{i=1}^{3} \theta_{i} A_{i}\right)\right\}<0$ holds then (9) holds with $\bar{P}=\left[\begin{array}{ll}P_{0} & 0_{n, 7 n}\end{array}\right]$ and $M$ defined in (16). The condition $\mathrm{He}\left\{P_{0}\left(A_{0}+\Sigma_{i=1}^{3} \theta_{i} A_{i}\right)\right\}<0$ is the condition based on quadratic stability. Thus, if the condition based on quadratic stability holds, then the condition of Theorem 1 always holds.

Conservatism reduction from quadratic stability is proved here for systems affine in three parameters. The same proof can be generalized for all parametrically affine models at the expense of recursive Schur complements, one for each parameter.

\section{2 Numerical Complexity vs. Conservatism}

In this subsection, we show that increasing parameterdependency of Lyapunov functions leads to less conservative analysis in exchange for increased numerical complexity.

For simplicity, we assume that the number of parameters is unity. However, we can easily extend the following result to the case that the number of parameters is $k(\geq 2)$.

Matrix $A(\theta)$ of (2) is given as follows.

$$
\begin{aligned}
& A(\theta)=\bar{A} \bar{\theta}_{n}=\left[\begin{array}{lllll}
A_{-l_{1}} & \cdots & A_{0} & \cdots & A_{m_{1}}
\end{array}\right] \bar{\theta}_{n} \\
& =\hat{A} \hat{\theta}_{n}=\left[\begin{array}{llllll}
A_{-l_{1}} & \cdots & A_{0} & \cdots & A_{m_{1}} & 0_{n}
\end{array}\right]\left[\begin{array}{lll}
\bar{\theta}_{n}^{T} & \theta_{1}^{m+1} I_{n}
\end{array}\right]^{T}
\end{aligned}
$$

We give the following assertion.

Proposition 2 If there exist matrices $\bar{P}$ and $M$ such that (9) holds for all vertices $\theta=\theta^{v_{1}}, \cdots, \theta^{v_{N}}$, then there always exist matrices $\hat{P} \in \mathcal{R}^{n \times n\left(l_{1}+m_{1}+1+1\right)}$ and $\hat{M} \in \mathcal{R}^{n\left(l_{1}+m_{1}+1\right) \times n\left(l_{1}+m_{1}+1+1\right)}$ such that (9), in which $\eta(\theta)$ is appropriately extended, holds for all vertices $\theta=$ $\theta^{v_{1}}, \cdots, \theta^{v_{N}}$. 
Proof 4 We assume that there exist matrices $\bar{P}$ and $M$ such that (9) holds for all vertices $\theta=\theta^{v_{1}}, \cdots, \theta^{v_{N}}$. We set $\hat{P}$ to be $\left[\begin{array}{ll}\bar{P} & 0_{n}\end{array}\right]$, and set $M$ in Theorem 1 to be

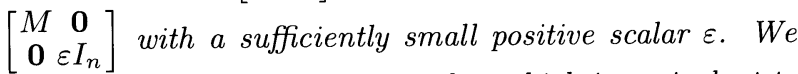
then obtain the following inequality which is equivalent to (9) with using $\hat{P}$ and $\hat{M}$.

$$
\left[\begin{array}{cc}
\operatorname{He}\left\{\bar{P}^{T} \bar{A}+\eta_{n}(\theta) M\right\} & {\left[\begin{array}{c}
\mathbf{0} \\
\varepsilon \theta_{1} I_{n}
\end{array}\right]} \\
{\left[\mathbf{0} \varepsilon \theta_{1} I_{n}\right]} & -2 \varepsilon I_{n}
\end{array}\right]<0,
$$

where $\eta_{n}(\theta)$ has a compatible size. After applying Schur complement to the above inequality, we obtain the following inequalities.

$$
\mathrm{He}\left\{\bar{P}^{T} \bar{A}+\eta_{n}(\theta) M\right\}+\left[\begin{array}{ll}
\mathbf{0} & \mathbf{0} \\
\mathbf{0} \frac{\varepsilon \theta_{1}^{2}}{2} I_{n}
\end{array}\right]<0, \varepsilon>0
$$

As the variation of $\theta_{1}$ is bounded, the first inequality of the above condition always holds with sufficiently small positive scalar $\varepsilon$ if the first term of the first inequality is negative definite, which is the assumption. Therefore there always exist $\hat{P}$ and $\hat{M}$ that satisfy (9). This completes the proof.

Conservatism reduction by increasing the power of parameters is proved here for systems with one parameter. The same proof can be generalized for all the considered models at the expense of recursive Schur complements, one for each power (negative and positive) of each parameter.

Proposition 2 shows that enlargement of the size of $\bar{P}$ leads to less conservative analysis, which was indicated in Remark 1. However, the enlargement of the size of $\bar{P}$ leads to the enlargement of the size of $M$, i.e. enlargement of the size of $\bar{P}$ leads to increased numerical complexity.

\section{Numerical Examples}

In this section, we show several numerical examples to demonstrate the effectiveness of our analysis methods.

\section{1 Polynomial Representation vs. Polytopic Representation}

We show an example that demonstrates the effectiveness of polynomial representation of LTIPD system in (2).

We consider the following system.

$$
\dot{x}=\left[\begin{array}{cc}
-(\theta+1 / \theta) & 1 \\
1 & -\theta
\end{array}\right] x, 0<\delta_{\min } \leq \theta \leq \delta_{\max }=100
$$

Note that this system is robustly stable for any $\delta_{\min }$. We minimize $\delta_{\min }$ under the condition that the system is assured to be robustly stable using our Theorem 1, Theorem 2 of 6 ), and Theorem 1 of 23) which is illustrated to be less conservative than Theorem 2 of 6 ) on numerical examples. When applying Theorem 2 of 6) and Theorem 1 of 23),
Table 1 Minimized $\delta_{\min }$

\begin{tabular}{|c|c|c|c|}
\multicolumn{1}{c|}{} & \multicolumn{3}{|c|}{ Theorem $1\left(l_{1}=m_{1}=1\right)$} \\
\hline & $\begin{array}{c}\text { Theorem 2 } \\
\text { of } 6)\end{array}$ & $\begin{array}{c}\text { Theorem } 1880 \times 10^{-4} \\
\text { of } 23)\end{array}$ & Gridding method \\
\hline $\begin{array}{c}\text { polytope } \\
\text { ABC }\end{array}$ & 0.9950 & 0.9950 & 0.9950 (40000 points) \\
\hline $\begin{array}{c}\text { polytope } \\
\text { ABDE }\end{array}$ & 0.5000 & 0.5000 & 0.5000 (80000 points) \\
\hline
\end{tabular}

Table 2 Minimized $\delta_{\min }$

\begin{tabular}{|c|c|}
\hline Theorem $1\left(l_{1}=1, m_{1}=1\right)$ & Biquadratic stability in 3$)$ \\
\hline $9.1880 \times 10^{-4}$ & 0.9950 \\
\hline
\end{tabular}

two polytopes are used to cover the variation of $\theta+1 / \theta$; polytope $\mathrm{ABC}$ and polytope ABDE in Fig. 1. Lyapunov functions used in Theorem 2 of 6) correspond to be cubically PDLFs with respect to the vertices; vertices A, B and $\mathrm{C}$ or vertices $\mathrm{A}, \mathrm{B}, \mathrm{D}$ and $\mathrm{E}$. Lyapunov functions used in Theorem 1 of 23) are set to be parameter-dependent up to second-order with respect to the vertices and their all by-products. The obtained $\delta_{\min }$ are given in Table 1. For comparison, we calculate $\delta_{\min }$ by fine gridding method, and the numbers of gridding points are denoted in parentheses. Considering that Theorem 2 of 6 ) and Theorem 1 of 23) produce the same stability margins as gridding method, these two methods are non-conservative for polytopically represented systems. However, Theorem 1 gives much tighter stability margin than Theorem 2 of 6 ) and Theorem 1 of 23). This result shows that stability analysis for polynomially parameter-dependent systems using polytopic approach is sometimes more conservative than using polynomial approach.

\section{2 Original Representation vs. Modified Rep- resentation}

In this subsection, we show that some modification of the original representation leads to conservatism.

We now consider the same example as in the previous subsection. Biquadratic stability proposed in 3) cannot be directly applied to this system, because this system is not affine with respect to $\theta$. To apply biquadratic stability to this system, we assume that the system has two parameters; $\theta$ and $\alpha:=1 / \theta$, whose deviations are respectively set as $\delta_{\min } \leq \theta \leq \delta_{\max }=100$ and $1 / \delta_{\max }=1 / 100 \leq \alpha \leq 1 / \delta_{\min }$. We minimize $\delta_{\min }$ under the condition that the system is assured to be robustly stable using biquadratic stability. The obtained $\delta_{\min }$ is given in Table 2. For comparison, we also show the obtained $\delta_{\min }$ using Theorem 1 with setting $l_{1}=m_{1}=1$, which was obtained in the previous subsection. For this example, Theorem 1 gives much tighter stability margin than biquadratic stability with assuming that the number of parameters to be two. This result shows that modifi- 
cation of system representation sometimes leads to conservatism.

\section{3 Randomly Generated Examples}

In this subsection, we demonstrate the effectiveness of our methods with randomly generated examples.

\subsubsection{Stability Analysis}

We consider the following four scenarios.

$\# 1: \quad A(\theta)=\Sigma_{i=0}^{2} \theta_{1}^{i} A_{i}, A_{i} \in \mathcal{R}^{2 \times 2}(i=0,1,2),\left|\theta_{1}\right| \leq$ $\delta$

$\# 2: \quad A(\theta)=\Sigma_{i=0}^{2} \theta_{1}^{i} A_{i}, A_{i} \in \mathcal{R}^{3 \times 3}(i=0,1,2),\left|\theta_{1}\right| \leq$ $\delta$

\#3: $\quad A(\theta)=A_{0}+\theta_{1} A_{1}, A_{i} \in \mathcal{R}^{4 \times 4}(i=0,1),\left|\theta_{1}\right| \leq \delta$

\#4: $\quad A(\theta)=\Sigma_{i=0}^{1} \Sigma_{j=0}^{1} \theta_{1}^{i} \theta_{2}^{j} A_{i j}, A_{i j} \in \mathcal{R}^{2 \times 2}(i, j=$ $0,1),\left|\theta_{1}\right| \leq \delta,\left|\theta_{2}\right| \leq \delta$

We generate 1000 examples for \#1 \#4. In all examples, $A_{0}$ or $A_{00}$ are generated as stable matrices, and all the elements of $A_{i}$ or $A_{i j}$ lie in $[-0.5 ; 0.5]$.

For each generated example, we maximize $\delta$ under the condition that the system is assured to be robustly stable using Theorem 1, and set obtained $\delta$ as $\delta_{\max }$. For comparison, we calculate the maximum value of $\delta$ using fine gridding method (1000 points for the deviation of $\theta_{1}$ in scenarios \#1 \#3 and each 200 points for the deviations of $\theta_{1}$ and $\theta_{2}$ in scenario \#4), and set obtained $\delta$ as $\delta_{\text {grid }}$. Table 3 shows the average values of $\delta_{\max } / \delta_{\text {grid }}$. We also give the number of decision variables as $N_{d v}$. Table 3 shows that Theorem 1 gives almost the exact stability margin, which demonstrates the effectiveness of Theorem 1.

\subsection{2 $\mathrm{H}_{2}$ Performance Analysis}

We consider the following scenario:

\#1: $\quad A(\theta)=\Sigma_{i=0}^{1} \Sigma_{j=0}^{1} \theta_{1}^{i} \theta_{2}^{j} A_{i j}, A_{i j} \in \mathcal{R}^{2 \times 2}(i, j=$ $0,1),\left|\theta_{1}\right| \leq \delta,\left|\theta_{2}\right| \leq \delta$, and other matrices $B(\theta) \in \mathcal{R}^{2 \times 2}$ and $C(\theta) \in \mathcal{R}^{2 \times 2}$ are also similarly generated.

We generate 1000 examples. In all examples, matrices $A_{00}$ are generated as stable matrices, and all the elements of $A_{i j}, B_{i j}$ and $C_{i j}$ lie in $[-0.5 ; 0.5]$.

For each generated example, we first calculate the stability margin $\delta$ by fine gridding method $\left(200^{2}\right)$ and set $\delta$ as $\delta_{\text {grid }}$, then calculate the upper bound of $H_{2}$ performance $\gamma_{2}$ by applying Theorems 2 and 5 with setting $\delta$ to be $0.8 \times \delta_{\text {grid }}$ and set $\gamma_{2}$ as $\gamma_{2_{\text {min }}}$. For comparison, we calculate the maximum value of $H_{2}$ performance $\gamma_{2}$ with setting $\delta$ to be $0.8 \times \delta_{\text {grid }}$ using fine gridding method $\left(200^{2}\right)$, and set $\gamma_{2}$ as $\gamma_{2_{\text {grid }}}$. Table 4 shows the average values of $\gamma_{2_{\min }} / \gamma_{2_{\text {grid }}}$. We also give the number of decision variables as $N_{d v}$. Table 4 shows that Theorem 5 gives more exact performance than Theorem 2, which demonstrates the less conservatism of Theorem 5 compared to
Table 3 Average values of $\delta_{\max } / \delta_{\text {grid }}$

\begin{tabular}{|c|c|c|c|c|c|c|}
\hline & & $\# 1$ & & $\# 2$ & & $\# 3$ \\
\hline $\begin{array}{l}\text { Theo } \\
\left(l_{1}=0,\right.\end{array}$ & $\begin{array}{l}\text { em } 1 \\
\left.m_{1}=1\right)\end{array}$ & - & & - & & $\begin{array}{l}.9986 \\
d v=52)\end{array}$ \\
\hline$\left(l_{1}=0\right.$, & $\begin{array}{l}\mathrm{em} 1 \\
\left.m_{1}=2\right)\end{array}$ & $\begin{array}{c}1.0000 \\
\left(N_{d v}=33\right)\end{array}$ & & $\begin{array}{l}9999 \\
v=72)\end{array}$ & $\left(N_{d}\right.$ & $\begin{array}{l}.0000 \\
v=126)\end{array}$ \\
\hline $\begin{array}{l}\text { Theo } \\
\left(l_{1}=0,\right.\end{array}$ & $\left.m_{1}=3\right)$ & $\begin{array}{c}1.0000 \\
\left(N_{d v}=60\right)\end{array}$ & & $\begin{array}{l}0000 \\
=132)\end{array}$ & & 8 \\
\hline & & & & \#4 & & \\
\hline & $\left(l_{i}=0\right.$, & $\begin{array}{l}\text { heorem } 1 \\
m_{i}=1, i=\end{array}$ & & $\begin{array}{c}0.999 \\
\left(N_{d v}=\right.\end{array}$ & & \\
\hline & $\left(l_{i}=0\right.$, & $\begin{array}{l}\text { heorem } 1 \\
m_{i}=2, i=\end{array}$ & & $\begin{array}{c}0.999 \\
\left(N_{d v}=\right.\end{array}$ & & \\
\hline
\end{tabular}

Table 4 Average values of $\gamma_{2 \min } / \gamma_{2}$ grid

\begin{tabular}{|c|c|}
\hline & $\# 1$ \\
\hline Theorem 2 & 1.0283 \\
$\left(l_{i}=0, m_{i}=1, i=1,2\right)$ & $\left(N_{d v}=301\right)$ \\
\hline Theorem 5 & 1.0032 \\
$\left(l_{i}=0, m_{i}=1, i=1,2\right)$ & $\left(N_{d v}=397\right)$ \\
\hline
\end{tabular}

Table 5 Average values of $\gamma_{\infty_{\text {min }}} / \gamma_{\infty_{\text {grid }}}$

\begin{tabular}{|c|c|}
\hline & $\# 1$ \\
\hline Theorem 3 & 1.0075 \\
$\left(l_{i}=0, m_{i}=1, i=1,2\right)$ & $\left(N_{d v}=205\right)$ \\
\hline Theorem 6 & 1.0053 \\
$\left(l_{i}=0, m_{i}=1, i=1,2\right)$ & $\left(N_{d v}=229\right)$ \\
\hline
\end{tabular}

Theorem 2.

\subsection{3 $H_{\infty}$ Performance Analysis}

We consider the following scenario:

\#1: $\quad A(\theta)=\Sigma_{i=0}^{1} \Sigma_{j=0}^{1} \theta_{1}^{i} \theta_{2}^{j} A_{i j}, A_{i j} \in \mathcal{R}^{2 \times 2}(i, j=$ $0,1),\left|\theta_{1}\right| \leq \delta,\left|\theta_{2}\right| \leq \delta$, and other matrices $B(\theta) \in$ $\mathcal{R}^{2 \times 2}, C(\theta) \in \mathcal{R}^{2 \times 2}$ and $D(\theta) \in \mathcal{R}^{2 \times 2}$ are also similarly generated.

We generate 1000 examples. In all examples, matrices $A_{00}$ are generated as stable matrices, and all the elements of $A_{i j}, B_{i j}, C_{i j}$ and $D_{i j}$ lie in $[-0.5 ; 0.5]$.

For each generated example, we first calculate the stability margin $\delta$ by fine gridding method $\left(200^{2}\right)$ and set $\delta$ as $\delta_{\text {grid }}$, then calculate the upper bound of $H_{\infty}$ performance $\gamma_{\infty}$ by applying Theorems 3 and 6 with setting $\delta$ to be $0.8 \times \delta_{\text {grid }}$ and set $\gamma_{\infty}$ as $\gamma_{\infty \text { min }}$. For comparison, we calculate the maximum value of $H_{\infty}$ performance $\gamma_{\infty}$ with setting $\delta$ to be $0.8 \times \delta_{\text {grid }}$ using fine gridding method $\left(200^{2}\right)$, and set $\gamma_{\infty}$ as $\gamma_{\infty \text { grid }}$. Table 5 shows the average values of $\gamma_{\infty_{\text {min }}} / \gamma_{\infty_{\text {grid }}}$. We also give the number of decision variables as $N_{d v}$. Table 5 shows that Theorem 6 gives more exact performance than Theorem 3, which demonstrates the less conservatism of Theorem 6 compared to Theorem 3.

These randomly generated examples demonstrate that our proposed methods produce almost the exact stability/performance analysis.

\section{4 Comparison with Existing Methods}

\subsubsection{Stability Analysis}

Consider now the following polytopic system borrowed from 6). 
Table 6 Maximized $\eta$ \begin{tabular}{|c|c|c|c|}
\hline Theorem 1 $\left(l_{i}=0, m_{i}=1, i=1,2\right)$ & Theorem 4 $\left(l_{i}=0, m_{i}=1, i=1,2\right)$ & Gridding method (1000 points $)$ & Theorem 2 of 6$)$ \\
\hline $3.5518\left(N_{d v}=132\right)$ & $3.5518\left(N_{d v}=156\right)$ & 3.5518 & $3.5518\left(N_{d v}=96\right)$ \\
\hline
\end{tabular}

Table 7 Maximized $\eta$

\begin{tabular}{|c|c|c|c|}
\hline $\begin{array}{c}\text { Theorem } 1 \\
\left(l_{i}=0, m_{i}=1, i=1,2,3\right)\end{array}$ & $\left(l_{i}=0, m_{i}=1, i=1,2,3\right)$ & Gridding method (40000 points) & Theorem 2 of 6$)$ \\
\hline $2.0605\left(N_{d v}=976\right)$ & $2.2238\left(N_{d v}=1136\right)$ & 2.2238 & $1.9306\left(N_{d v}=204\right)$ \\
\hline
\end{tabular}

$$
A(\theta)=\Sigma_{i=1}^{2} \theta_{i}\left(A_{0}+\eta A_{i}\right),
$$

where $\theta^{v_{1}}=\left[\begin{array}{ll}1 & 0\end{array}\right]^{T}, \theta^{v_{2}}=\left[\begin{array}{ll}0 & 1\end{array}\right]^{T}$, and $A_{i}(i=0,1,2)$ are defined as follows:

$$
A_{0}=\left[\begin{array}{ccc}
-2.0 & 1.0 & -1.0 \\
2.5 & -3.0 & 0.5 \\
-1.0 & 1.0 & -3.5
\end{array}\right], A_{1}=\left[\begin{array}{ccc}
-0.7 & -0.5 & -2.0 \\
-0.8 & 0.0 & 0.0 \\
1.5 & 2.0 & 2.4
\end{array}\right], A_{2}=-A_{1} .
$$

Table 6 shows the maximized $\eta$ under the condition that the system is assured to be robustly stable. For comparison, we show the number of decision variables as $N_{d v}$. Theorem 1 with setting $l_{i}=0, m_{i}=1(i=1,2)$, Theorem 4 with setting $l_{i}=0, m_{i}=1(i=1,2)$, and Theorem 2 of 6 ) all give the exact stability margin. However, the numbers of decision variables in our methods are larger than that in Theorem 2 of 6 ). Thus, Theorem 2 of 6 ) is effective compared to our methods in terms of numerical complexity.

We next consider the following polytopic system borrowed from 6 ), which was originally published in 17).

$$
A(\theta)=\Sigma_{i=1}^{3} \theta_{i}\left(A_{0}+\eta A_{i}\right),
$$

where $\theta^{v_{1}}=\left[\begin{array}{lll}1 & 0 & 0\end{array}\right]^{T}, \theta^{v_{2}}=\left[\begin{array}{lll}0 & 1 & 0\end{array}\right]^{T}, \theta^{v_{3}}=\left[\begin{array}{lll}0 & 0 & 1\end{array}\right]^{T}$, and $A_{i}(i=0, \cdots, 3)$ are defined as follows:

$$
\begin{aligned}
& A 0=\left[\begin{array}{cccc}
-2.4 & -0.6 & -1.7 & 3.1 \\
0.7 & -2.1 & -2.6 & -3.6 \\
0.5 & 2.4 & -5.0 & -1.6 \\
-0.6 & 2.9 & -2.0 & -0.6
\end{array}\right], A_{1}=\left[\begin{array}{cccc}
1.1 & -0.6 & -0.3 & -0.1 \\
-0.8 & 0.2 & -1.1 & 2.8 \\
-1.9 & 0.8 & -1.1 & 2.0 \\
-2.4 & -3.1 & -3.7 & -0.1
\end{array}\right] \text {, } \\
& A_{2}=\left[\begin{array}{cccc}
0.9 & 3.4 & 1.7 & 1.5 \\
-3.4 & -1.4 & 1.3 & 1.4 \\
1.1 & 2.0 & -1.5 & -3.4 \\
-0.4 & 0.5 & 2.3 & 1.5
\end{array}\right], A_{3}=\left[\begin{array}{cccc}
-1.0 & -1.4 & -0.7 & -0.7 \\
2.1 & 0.6 & -0.1 & -2.1 \\
0.4 & -1.4 & 1.3 & 0.7 \\
1.5 & 0.9 & 0.4 & -0.5
\end{array}\right] \text {. }
\end{aligned}
$$

Table 7 shows the maximized $\eta$ under the condition that the system is assured to be robustly stable. For this example, the method of 17) gives the exact stability margin, and Theorem 2 of 6 ) does not give the exact stability margin. Theorem 1 with setting $l_{i}=0, m_{i}=1(i=1,2,3)$ does not give the exact stability margin. However, Theorem 4 with setting $l_{i}=0, m_{i}=1(i=1,2,3)$ gives the exact stability margin.

This example demonstrates the effectiveness of our methods compared to Theorem 2 of 6 ).

\subsection{2 $H_{\infty}$ Performance Analysis}

Consider the following polytopic system borrowed from $6)$.

$$
\left[\begin{array}{l|l}
A(\theta) & B(\theta) \\
\hline C(\theta) & D(\theta)
\end{array}\right]=\Sigma_{i=1}^{3}\left[\frac{\theta_{i} A_{i} \mid \theta_{i} B_{i}}{\theta_{i} C_{i} \mid \theta_{i} D_{i}}\right],
$$

where $A_{i}(i=1,2,3)$, etc. are defined as follows.
Table 8 Upper bound of $H_{\infty}$ performance

\begin{tabular}{|c|c|c|}
\hline $\begin{array}{c}\text { Theorem 3 } \\
\left(l_{i}=0, m_{i}=1, i=1,2,3\right)\end{array}$ & $\begin{array}{c}\text { Gridding method } \\
(40000 \text { points })\end{array}$ & $\begin{array}{c}\text { Theorem 5 } \\
\text { of } 6)\end{array}$ \\
\hline $\begin{array}{c}1.8716 \\
\left(N_{d v}=945\right)\end{array}$ & 1.8715 & $\left(N_{d v}=106\right)$ \\
\hline
\end{tabular}

Table 9 Upper bound of $H_{\infty}$ performance

\begin{tabular}{|c|c|c|}
\hline $\begin{array}{c}\text { Theorem 3 } \\
\left(l_{i}=0, m_{i}=1, i=1,2\right)\end{array}$ & $\begin{array}{c}\text { Gridding method } \\
(1000 \text { points })\end{array}$ & $\begin{array}{c}\text { Theorem 5 } \\
\text { of } 6)\end{array}$ \\
\hline 1.0140 & 1.0140 & $\begin{array}{c}1.0258 \\
\left(N_{d v}=91\right)\end{array}$ \\
\hline
\end{tabular}

$$
\begin{aligned}
& A_{1}=\left[\begin{array}{ccc}
-0.5008 & -0.4073 & 0.0336 \\
0.2194 & 0.3801 & 0.5698 \\
-0.4972 & -0.6033 & -0.2826
\end{array}\right], \\
& A_{2}=\left[\begin{array}{ccc}
-0.1603 & -0.1433 & -0.3133 \\
-0.1536 & -1.254 & 0.3023 \\
0.5079 & 0.2159 & -0.1128
\end{array}\right], \\
& A_{3}=\left[\begin{array}{ccc}
-0.2436 & 0.1515 & -0.1856 \\
-0.5742 & 0.0975 & 0.6841 \\
-0.3823 & -0.4388 & -0.9544
\end{array}\right], \\
& B_{1}=\left[\begin{array}{c}
0.2190 \\
-0.6587 \\
0.7503
\end{array}\right], B_{2}=B_{1}, B_{3}=B_{1}, \\
& C_{1}=\left[\begin{array}{ll}
-0.08900 .3838-0.0374
\end{array}\right], C_{2}=C_{1}, C_{3}=C_{1}, \\
& D_{1}=-0.4786, D_{2}=D_{1}, D_{3}=D_{1}
\end{aligned}
$$

Table 8 shows the minimized upper bound of $H_{\infty}$ performance. For this example, Theorem 3 and Theorem 5 of 6 ) both give the exact $H_{\infty}$ performance analysis. However, our method needs more numerical complexity than Theorem 5 of 6 ). Therefore, our method is not so effective as Theorem 5 of 6 ) in terms of numerical complexity.

We finally consider the following polytopic system borrowed from 6 )

$$
\left.\left[\frac{A(\theta) \mid B(\theta)}{C(\theta)}\right] \overline{D(\theta)}\right]=\Sigma_{i=1}^{2}\left[\frac{\theta_{i} A_{i} \mid \theta_{i} B_{i}}{\theta_{i} C_{i} \mid \theta_{i} D_{i}}\right],
$$

where $A_{i}(i=1,2)$, etc. are defined as follows.

$$
\begin{aligned}
& A_{1}=\left[\begin{array}{ccc}
-0.4820 & 0.2475 & 0.5523 \\
0.6564 & -0.0541 & 0.6678 \\
0.4631 & -0.8679 & -0.7465
\end{array}\right], \\
& A_{2}=\left[\begin{array}{ccc}
-0.3280 & -0.4585 & -0.0199 \\
0.2639 & 0.1258 & -0.0179 \\
-0.0156 & 0.1079 & -0.5112
\end{array}\right], \\
& B_{1}=\left[\begin{array}{c}
-0.7089 \\
0.0738 \\
0.5886
\end{array}\right], B_{2}=\left[\begin{array}{c}
0.0118 \\
-0.5969 \\
0.0295
\end{array}\right] \text {, } \\
& C_{1}=[0.31260 .3343-0.3119], C_{2}=C_{1} \text {, } \\
& D_{1}=0.2713, D_{2}=D_{1}
\end{aligned}
$$

Table 9 shows the minimized upper bound of $H_{\infty}$ performance. For this example, Theorem 5 of 6 ) does not give the exact performance, however, Theorem 3 gives almost the exact $H_{\infty}$ performance analysis. Therefore, our 
method is more effective than Theorem 5 of 6 ).

\section{Conclusions}

We propose general formulations for robust stability, $\mathrm{H}_{2}$ performance, and $H_{\infty}$ performance analysis for linear time-invariant uncertain systems, whose state-space matrices are parameter-dependent in negative as well as positive power series of parameters, using Lyapunov functions which are parameter-dependent in negative as well as positive power series of parameters. Although our formulations are only sufficient conditions for the original analysis problems because we apply slack variable approach. However, randomly generated examples and several examples borrowed from existing papers demonstrate the effectiveness of our methods compared to existing methods.

\section{Acknowledgment}

The authors gratefully thank anonymous reviewers for their fruitful comments.

\section{References}

1) M. C. de Oliveira, J. Bernussou and J. C. Geromel: A New Discrete-Time Robust Stability Condition, Systems \& Control Letters, 37-4, 261/265 (1999)

2) A. T. Neto: Parameter Dependent Lyapunov Functions for a Class of Uncertain Linear Systems: and LMI Approach, Proc. CDC, 2341/2346 (1999)

3) A. Trofino and C. E. de Souza: Biquadratic Stability of Uncertain Linear Systems, IEEE Trans. Automatic Control, 46-8, 1303/1307 (2001)

4) Y. Ebihara and T. Hagiwara: A Dilated LMI Approach to Robust Performance Analysis of Linear Time-Invariant Uncertain Systems, Automatica, 41-11, 1933/1941 (2005)

5) M. Sato: Robust Performance Analysis of Linear TimeInvariant Parameter-Dependent Systems using HigherOrder Lyapunov Functions, Proc. ACC, 615/620 (2005)

6) Y. Ebihara, D. Peaucelle, D. Arzelier and T. Hagiwara: Robust Performance Analysis of Linear TimeInvariant Uncertain Systems by Taking Higher-Order Time-Derivatives of the State, Proc. CDC, 5030/5035 (2005)

7) D. Peaucelle, D. Arzelier, O. Bachelier and J. Bernussou: A New Robust $\mathcal{D}$-Stability Condition for Real Convex Polytopic Uncertainty, Systems \& Control Letters, 40-1, $21 / 30(2000)$

8) Y. Ebihara, D. Peaucelle, D. Arzelier and T. Hagiwara: Robust $\mathrm{H}_{2}$ Performance Analysis of Uncertain LTI Systems via Polynomially Parameter-Dependent Lyapunov Functions, IFAC Robust Control Design (2006)

9) D. Peaucelle, Y. Ebihara, D. Arzelier and T. Hagiwara: General Polynomial Parameter-Dependent Lyapunov Functions For Polytopic Uncertain Systems, Proc. MTNS, 2238/2242 (2006)

10) R. C. L. F. Oliveira and P. L. D. Peres: Less Conservative LMI Conditions for $\mathcal{D}$-stability, Proc. $C D C, 5149 / 5154$ (2003)

11) V. J. S. Leite and P. L. D. Peres: An Improved LMI Condition for Robust $\mathcal{D}$-Stability of Uncertain Polytopic
Systems, IEEE Trans. Automatic Control, 48-3, 500/504 (2003)

12) R. C. L. F. Oliveira and P. L. D. Peres: LMI Conditions for the Existence of Polynomially Parameter-Dependent Lyapunov Functions Assuring Robust Stability, Proc. $C D C$, $1660 / 1665$ (2005)

13) Y. Oishi: A Region-Dividing Approach to Robust Semidefinite Programming and Its Error Bound, Proc. ACC, $123 / 129(2006)$

14) R. C. L. F. Oliveira and P. L. D. Peres: LMI Conditions for Robust Stability Analysis Based on Polynomially Parameter-Dependent Lyapunov Functions, Systems \& Control Letters, 55-1, 52/61 (2006)

$15)$ D. Peaucelle and D. Arzelier: Robust Performance Analysis with LMI-Based Methods for Real Parametric Uncertainty via Parameter-Dependent Lyapunov Functions, IEEE Trans. Automatic Control, 46-4, 624/630 (2001)

16) T. Iwasaki and G. Shibata: LPV System Analysis via Quadratic Separator for Uncertain Implicit Systems, IEEE Trans. Automatic Control, 46-8, 1195/1208 (2001)

17) G. Chesi, A. Garulli, A. Tesi and A. Vicino: Robust Stability of Polytopic Systems via Polynomially ParameterDependent Lyapunov Functions, Proc. CDC, 4670/4675 (2003)

18) G. Chesi, A. Garulli, A. Tesi and A. Vicino: Polynomially Parameter-Dependent Lyapunov Functions for Robust $H_{\infty}$ Performance Analysis, Proc. of the 16th IFAC World Congress (2005)

19) P. A. Bliman: A Convex Approach to Robust Stability for Linear Systems with Uncertain Scalar Parameters, SIAM J. Control and Optimization, 42-6, 2016/2042 (2004)

20) D. Coutinho, A. Trofino and M. Fu: Guaranteed Cost Control of Uncertain Nonlinear Systems via Polynomial Lyapunov Functions, IEEE Trans. Automatic Control, 47-9, $1575 / 1580$ (2002)

21) E. Feron, P. Apkarian and P. Gahinet: Analysis and Synthesis of Robust Control Systems via ParameterDependent Lyapunov Functions, IEEE Trans. Automatic Control, 41-7, 1041/1046 (1996)

22) T. Iwasaki and R. E. Skelton: All Controllers for the General $H_{\infty}$ Control Problem: LMI Existence Conditions and State Space Formulas, Automatica, 30-8, 1307/1317 (1994)

23) M. Sato and D. Peaucelle: Robust Stability/Performance Analysis for Linear Time-Invariant Polytopically ParameterDependent Systems using Polynomially ParameterDependent Lyapunov Functions, Proc. CDC, 5814/5819 (2006)

Masayuki Sato (Member)

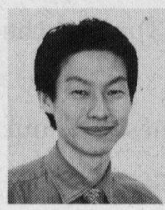

Masayuki Sato received the B.E. and M.E. degrees in aerospace engineering from Nagoya University, Nagoya, Japan, in 1995 and 1997, respectively. Since 1997, he has been with National Aerospace Laboratory (now, Japan Aerospace Exploration Agency) as a researcher. His research interests include robust/gain-scheduled controller design and their applications to flight controllers. 


\section{Dimitri Peaucelle}

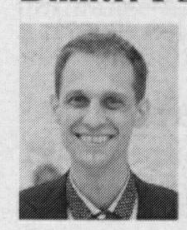

Dimitri Peaucelle received the Engineer's degree from the Ecole Centrale de Lille, France, in 1997 and Master's degree from the University of Science and Technology of Lille, in 1997. He received the Ph.D. degree from the Paul Sabatier University, Toulouse, France, in 2000. Since 2001, he holds a position at LAASCNRS. His research interests are robust multiperformance control, low-order control design, and optimization related to semi-definite programming. 